\title{
Design and development of laser detection device for bottled biological
}

\section{products}

\author{
Lijian Zhang ${ }^{1, a}$, Mei Lin ${ }^{1, b}$, Tingting Wang ${ }^{2, c}$ \\ ${ }^{1}$ College of Electrical Engineering, Binzhou Polytechnic,Shandong,256600,China \\ ${ }^{2}$ Bohai Piston Co., Ltd, Binzhou,Shandong,256600,China \\ axianruibrother@163.com, b41350992@qq.com, '1653226361@qq.com
}

Keywords: Detection, Point light source, Polarized light location, Control

\begin{abstract}
Point source of light across the bottle detection technology is a rapid detection technology of modern biotechnology, pharmaceutical, chemical, food and other products, fast test bottle. The testing instrument comprises a sample bottles and a conversion device, light source system and polarized light measuring device, the sample bottle optical position adjusting device, light intensity, displacement detection device and control system six parts. The optical target bottle samples obtained directly without opening the bottle under the circumstances, to ascertain the quality of bottled biological products.
\end{abstract}

\section{Introduction}

At present at home and abroad is not effective "biological products" rapid identification method, although there are other chemical identification methods, polygenetic specific difference and require the destruction test and discarded. Using optical every bottle of detection technology for the rapid identification of drug research does not see more, uses a laser to detect technology every bottle of rapid identification of biological products research at present there is no for use laser detection of human albumin to open up a new way.

Working principle

The identification of biological products - human blood albumin technology by laser penetrating glass bottle is the use of laser penetrating glass bottle. The interference effect of liquid in the bottle is detected, and the purpose is reached.. According to the project requirements, the optical properties of human serum albumin were studied.. In order to determine human serum albumin optical characteristics index, first of all according to the characteristics of the structure of human serum albumin and absorption of light, interference effect characteristic carries on the theoretical analysis, and according to the results of the analysis using existing instrumentation test analysis of human serum albumin solution, and eventually optimize selected is representative, specificity and easy detection of the optical characteristics. Then to preferred optical characteristics index, were tested and analyzed using the indexes of optical instrument with a single parameter test functions for the optimum person blood albumin solution, at the same time, the possible human serum albumin adulterants comparative experiment was carried out to verify the reliability of the optical characteristics and specificity, ultimately for fast every bottle of identification of human blood albumin determine specific detection index. At the same time, for the further implementation of empty and load spectra difference calculations have shown that, spectral function of a / D conversion, multidimensional curve fitting operations and human serum albumin fast every bottle of instrument to identify the specific design, to realize the nondestructive technology for rapid identification of biological products from the scene "to complete the basic research.

By point source, eliminating the interference of the bottle wall of incident light; the light, electric detection system and a sample bottle to rotate together, detection in the process of carrying liquid bottle and empty bottles and incident spot position remained relatively consistent so as to eliminate the interference of the empty bottle part. By using light path installation optical measuring device 
and photoelectric displacement measuring device for the simultaneous detection of load level, with the empty bottles a absorbance, optical rotation, refractive index, optical index. Through the system difference operation treatment, in bottles are obtained directly from the sample bottle of the optical index, in order to ascertain the quality of the bottle.

\section{Mechanical component}

Using a point source every bottle of detection technology, its characteristics is that the sample bottle optical position adjusting device (105) the sample bottle and a point light source system (103) and polarized light microscope automatic rotating device (104), emergent light intensity, displacement detection device (106) remain in the same line, the sample bottles, load level switching device (102) makes the point light source system (103) from the spot and the sample bottles in the empty bottle and load level remains same point, in order to eliminate the bottle wall of incident light interference, and the optical path with polarized light to measure device (104), at the same time, completes the bottle like liquid rotation, refractive index and optical absorbance determination.



Fig.1 equipment section description

System about the system, to observe the position of the sample bottles, load level switching device (102) is mainly composed of a rotating cabin (201), left and right rotation support hollow shaft (202), sample bottle hatch (203), sample bottle optical window (204); the sample bottle hatch (203) and sample bottle light path location observation window (204) in rotating cabin (201) top left and right rotary support hollow shaft (202) connected to the left and right sides of the hollow shaft gear and the fixed bracket (101) rotary poking device (205) dial wheel meshed rotating cabin (201) internal rotation support hollow shaft (202) hole and the fixing bracket (101) cavity are communicated with each other.

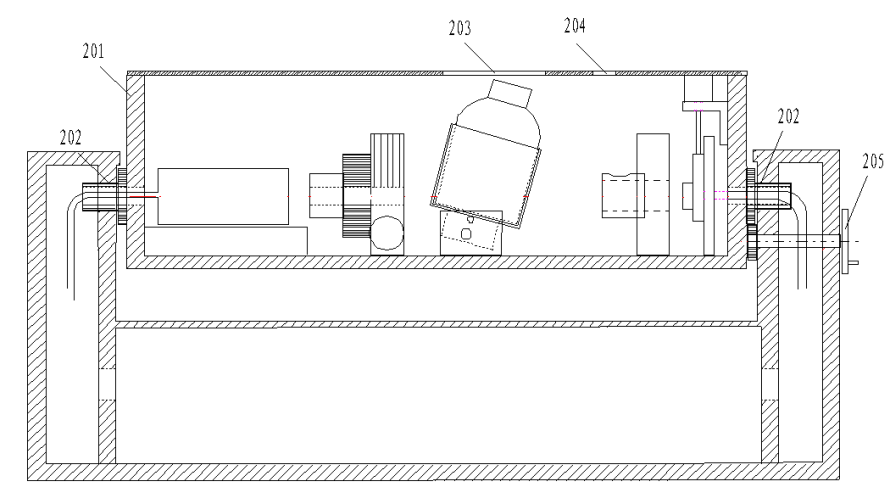

Fig.2 equipment section description

Point light source system (103) monochromatic light conversion part (301), conductive fiber (302), point source conversion part (303), monochromatic light conversion part (301) is located in the system fixed bracket (101) at the bottom left, point source conversion part (303) in rotating 
cabin (201), conductive fiber (302) through the left and right rotary support hollow shaft (202) hole and two were connected to a point light source system (103) mainly is composed of a luminous body, a diffraction grating, a lens, a filter, a collimator, optical fiber, optical bar is composed, of which the luminous body for full wavelength long-life lamp or diode laser or LED cold light source, the use of wavelength can be adjusted.

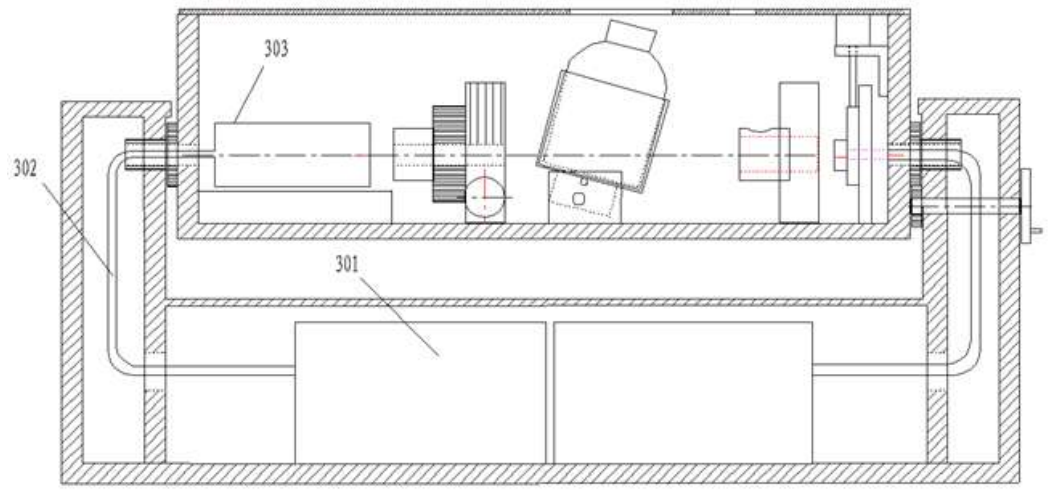

Fig.3 equipment section description

The polarized determination device (104) is mainly composed of a front polarizer (401), electric rotary disc (402), rear polarizer (403), pre polarizer (401) embedded in the electric rotary disc (402) center hole, electric rotary disc (402) and rear polarizer (403) are respectively fixed on both sides of the rotating cabin (203) at the bottom of the optical path of the sample bottle position adjustment device (106), electric rotary disc (402) can also be located in the rear polarizer (403) position matched with.

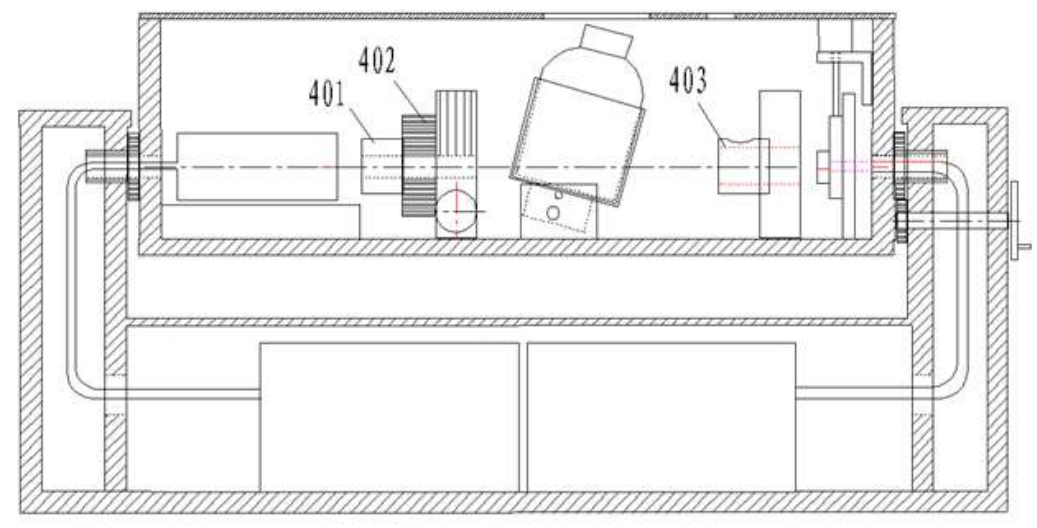

Fig.4 equipment section description

Shift left shift right, scattered in the shift left and right shift of the sample bottle optical position adjusting device (105) is mainly composed of a left and a right fixed bracket (501), a bottle clamping plate inclination adjusting frame (502), bottle about splint (503), a bottle clamp screw (504), a bottle clamp screw (505); bottle splint inclination adjusting frame (502) located in the left and right fixing bracket (501) inside the bottle around splint (503) half bottle splint inclination adjusting frame (502) inside the bottle around splint (503) is arranged on the shaft hole and screw the bottle clamp screw (504) and a bottle clamping plate screw (505) are respectively inserted in the shaft hole and a screw hole. 


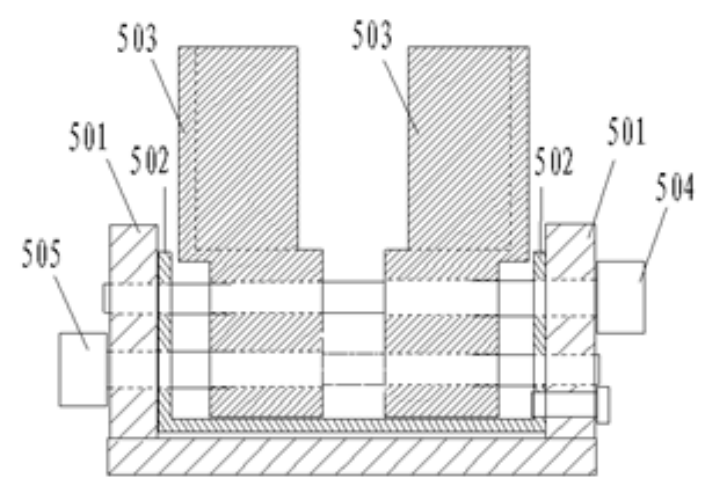

Fig.5 equipment section description

Characterized by, the control system (107) in systems with a fixed bracket (101) at the bottom, mainly by the motion control system, electrical control system, temperature control system, a photoelectric detection system, a power supply device is formed.

\section{Control system constitution}

\section{Electronic components}

This system mainly by the Beijing Kunlun Tongtai touch screen, PLC, all photoelectric sensor, step and stepper motor drive and other equipment.

The PLC receives a signal, photoelectric sensor control of coordinated motion of two stepper motor, to achieve the purpose of detecting albumin. The control of a stepper motor drive linear motion system, moving back and forth two times, find the strongest test point light source.

In the man-machine interface, the system parameters, motion control, curve display, data storage and sound and light alarm are set up.. Because of the complexity of PLC programming, data analysis and collection to PLC, serial communication program is sent to the touch screen, and the data analysis program is written in the touch screen script..

\section{Equipment selection}

The device has $1024 \mathrm{~V}$ powered photoelectric switch, and 1 start button, 1 stop button and 1 stop button, can be directly connected with the PLC input side. The output part mainly includes 2 stepper motor control and 1 sound and sound alarm.

According to input and output their points and signal type, select the Luoyang blue fly YH-0A an integral type programmable controller, the models have 16 bit input 14 bit output, the CPU module integrated support Modbus RTU protocol of RS485 serial port, convenient and MCGS connection, without the need to write drivers. Select the 1 block positioning expansion module YF0A-PTO, the module provides 2 axis positioning control output (pulse + direction), each axis has 1 way origin capture input.

\section{Experimental methods and technical route}

1.According to the structure of human serum albumin, the theoretical indications for optical identification are determined.

And, so as to choose the suitable for laser detection of human serum albumin in differential theory of optical characteristics index by data query (where poor) understand albumin biological products of molecular structure and optical characteristics.

2.Using the optical test analysis to optimize the combination and determine the target optical characteristic group

General optical identification, the majority is targeted for one-way optical characteristics, but only a single feature of the identification of indicators, it is difficult to determine the accuracy of the material measured. Based on this principle, for biological products in ultraviolet, visible and infrared region absorption spectra analysis and optimized with significance in differentiating 
multiple optical characteristics index and combinatorial optimization, to determine the value of the optical characteristics of the target.

3.according to the target optical characteristics, the verification experiment is carried out.

The first experimental analysis of human serum albumin standard solution, to select the final out with representative, specificity and easy detection of optical characteristics index group, with single index function test instrument, test certificate to optimize human serum albumin solution of the optical indicators. Then the albumin optical index analysis of listed human serum albumin were tested. The reliability and specificity of the potential of the human serum albumin artifact were compared with the test to verify the reliability and specificity of the selected optical characteristics.. Finally, the identification of the optical characteristics of albumin by laser detection technology is the target of the identification of the albumin in the biological products.

4.according to the optical index, the theoretical design of the separation of the albumin by laser is accomplished.

According to the glass bottle structure characteristics and an optical conduction Snell's law, of laser in the bottle, a liquid conductive trace analysis, using modern optical detection technology, electronic technology, using laser vibration theory design route - bottle inspection of biological products of human serum albumin.

\section{References}

[1]Yu X,Liang X,HyyppaJ,etal.Stem biomass estimation based on stem reconstruction from terrestrial laser scanning point clouds[J]. Remote Sensing Letters, 2013,4:344 - 353.

[2] $\mathrm{Xu}$ Gen.The application of PLC simulation software in the equipment management [J] , equipment management and maintenance, 2009 (7): 19-20.

[3] Li Zhongcheng. Research and design of environmental monitoring system based on wireless sensor networks [J]. Computer measurement and control, 2008, 16 (7): 929- 931.

[4]ZhaoanYu, zeTian. Reliability research of [D] and real time WinCE software based on embedded industrial control system. Master's thesis of Northwestern University.

[5] Shimin Wang. Design of [J] automatic equipment andmicro powder. Mechanical research and application, 2013(2): 123-126. 